\title{
Relative stability of negative and positive trions in model symmetric quantum wires
}

\author{
B. Szafran, ${ }^{1,2}$ T. Chwiej, ${ }^{2}$ F. M. Peeters, ${ }^{1}$ S. Bednarek, ${ }^{2}$ and J. Adamowski ${ }^{2}$ \\ ${ }^{1}$ Departement Fysica, Universiteit Antwerpen (Campus Drie Eiken), Universiteitsplein 1, B-2610 Antwerpen, Belgium \\ ${ }^{2}$ Faculty of Physics and Applied Computer Science, AGH University of Science and Technology, al. Mickiewicza 30, \\ 30-059 Kraków, Poland
}

(Received 11 January 2005; published 6 June 2005)

\begin{abstract}
Binding energies of negative $\left(X^{-}\right)$and positive trions $\left(X^{+}\right)$in quantum wires are studied for strong quantum confinement of carriers which results in a numerical exactly solvable model. The relative electron and hole confinement have a strong effect on the stability of trions. For equal hole and electron confinement, $X^{+}$is more stable but a small imbalance of the particle confinement towards a stronger hole confinement, e.g., due to its larger effective mass, leads to the interchange of $X^{-}$and $X^{+}$recombination lines in the photoluminescent spectrum as was recently observed experimentally. In case of larger $X^{-}$stability, a magnetic field oriented parallel to the wire axis leads to a stronger increase of the $X^{+}$binding energy resulting in a crossing of the $X^{+}$ and $X^{-}$lines.
\end{abstract}

DOI: $10.1103 /$ PhysRevB.71.235305

PACS number(s): 68.65.La, 71.35.Pq

\section{INTRODUCTION}

Trions are charged exciton complexes formed when an electron or a hole is bound ${ }^{1}$ to a neutral exciton $(X)$. The binding energies of the complexes are very small in bulk, but they are substantially enhanced in structures of reduced dimensionality, i.e., in quantum wells ${ }^{2-8}$ and quantum wires. $^{9-11}$

Due to the larger effective mass of the hole, in bulk ${ }^{12}$ as well as in strictly two-dimensional confinement ${ }^{3}$ the binding energy of positive trions $\left(X^{+}\right)$is larger than the negative trion $\left(X^{-}\right)$binding energy. However, in quantum wells the observed $^{5} X^{-}$and $X^{+}$binding energies are nearly equal, which is explained ${ }^{4,5}$ by a stronger hole confinement within the quantum well enhancing the hole-hole interaction. The magnetic field perpendicular to the plane of confinement enhances more strongly the $X^{-}$stability leading to a crossing of $X^{-}$and $X^{+}$binding energies. ${ }^{13,14}$ For trions localized on a defect of the quantum well potential $X^{-}$can become more stable than $X^{+}$even without the presence of an external magnetic field. ${ }^{15}$ The combined quantum well and defect confinement creates a three-dimensional potential similar to a quantum dot. In quantum dots the confinement-related hole-hole interaction enhancement leads to the interchange of the order of the $X^{-}$and $X^{+}$recombination lines in the photoluminescence (PL) spectrum already for quantum dot diameters as large as 24 donor Bohr radii. ${ }^{16}$ For smaller dots the $X^{+}$line was predicted to become even more energetic ${ }^{16,17}$ than the $X$ line, which was recently confirmed experimentally. ${ }^{18} \mathrm{In}$ coupled dots ${ }^{17,19}$ this effect leads to the ground-state dissociation of $X^{+},{ }^{17,19}$ for which the holes in the ground state occupy different dots.

The present work is motivated by a recent experimental study ${ }^{11}$ of positive and negative exciton trions in V-groove $\mathrm{GaAs} / \mathrm{AlGaAs}$ quantum wires. The negative trion was found to be distinctly more stable than $X^{+}$(binding energies of $X^{-}$ and $X^{+}$were determined as 4.2 and $2.9 \mathrm{meV}$, respectively). Here, we indicate that the observed ${ }^{11}$ order of $X^{-}$and $X^{+}$ energy lines may be a consequence of modifications of the interactions due to a stronger hole confinement. In a previous theoretical study ${ }^{9}$ of trions in quantum wires $X^{+}$was found to be more stable than $X^{-}$, which was obtained in the case of equal hole and electron confinement. A crossing of $X^{-}$and $X^{+}$ PL lines as functions of the wire width has previously been obtained in a quantum Monte Carlo study ${ }^{10}$ of a quantum wire with a square well confinement potential. In this paper we focus on the effect due to different electron and hole confinement leading to modifications of the effective interparticle interactions. We study the correlations between electrons and holes and consider the effect of a magnetic field oriented parallel to the quantum wire. The study of the stability of the trions is performed as function of the electron and hole confinement instead of dimensions of the wire. It has been demonstrated ${ }^{20}$ that in realistic quantum wires with strong confinement the binding energy of neutral excitons is governed by a size dependent parameter independent of the shape and composition of the wire.

The stronger hole confinement results from its weak penetration into the barrier material due to its larger effective mass than the electron band mass. For the spillover of the electron wave function out of the quantum wire, recently observed in self-assembled InAs/InP quantum wires, ${ }^{21}$ the ratio of the electron to hole confinement can in principle be arbitrarily large. ${ }^{21}$ However, in the following we show that even a small enhancement of the hole confinement changes the order of the $X^{-}$and $X^{+}$PL recombination lines.

For the purpose of the present study we apply the single band model for the hole and consider a harmonic oscillator confinement potential in the directions perpendicular to the wire, referred to as "lateral" in the following. The present model does not account for the interface between the wire and barrier materials, so the effective mass discontinuity and dielectric constant mismatch are neglected. These effects usually strengthen the electron-hole interaction and weaken the penetration of the wave functions into the barrier. They are, however, of a secondary importance for $\mathrm{GaAs} / \mathrm{AlGaAs},{ }^{22}$ InAs/InP, ${ }^{23}$ and $\mathrm{CdTe} / \mathrm{ZnTe}^{24}$ quantum wires. Note that, the present modelling is inapplicable to the 
free-standing quantum wires, where the image charge effect is extremely strong. ${ }^{25}$

We assume that the lateral confinement is strong, so that only the lowest subband for the electron and hole is occupied. This assumption allows for a reduction of the Schrödinger equation to an effective two-dimensional form. Usually the solution of the trion eigenequations is very challenging and requires extensive variational calculations $^{2-4,6,7,12,16,17}$ or application of the quantum Monte Carlo methods. ${ }^{10,15}$ The present problem is unique in the sense that it allows for an exact inclusion of the interparticle correlations.

The paper is organized as follows: the next section contains the theory, the results are given in Sec. III, the conclusion and summary are presented in Sec. IV.

\section{THEORY}

We adopt the donor units, i.e., donor Bohr radius $a_{d}=4 \pi \epsilon_{0} \epsilon \hbar^{2} / m_{e} e^{2}$ for the unit of length and twice the donor Rydberg $2 R_{d}=\hbar^{2} / m_{e} a_{d}^{2}$ as the unit of the energy, where $m_{e}$ is the band electron effective mass and $\epsilon$ is the dielectric constant. In these units, the Hamiltonian for a single electron in a quantum wire with harmonic oscillator lateral confinement has the form

$$
H_{e}=-\frac{1}{2} \frac{\partial^{2}}{\partial z_{e}^{2}}+H_{e}^{l},
$$

with the lateral Hamiltonian

$$
H_{e}^{l}=-\frac{1}{2}\left(\frac{\partial^{2}}{\partial x_{e}^{2}}+\frac{\partial^{2}}{\partial y_{e}^{2}}\right)+\frac{1}{2 l_{e}^{4}}\left(x_{e}^{2}+y_{e}^{2}\right),
$$

where $l_{e}$ is the length of the harmonic oscillator confinement for the electron. The ground-state wave function of the Hamiltonian (2) is $\Psi_{e}=\exp \left[-\left(x^{2}+y^{2}\right) / 2 l_{e}^{2}\right] / l_{e} \sqrt{\pi}$ with the energy eigenvalue $E_{e}=1 / l_{e}^{2}$. In the adopted single-band approximation the hole ground-state wave function $\left(\Psi_{h}\right)$ of the lateral confinement has the form of $\Psi_{e}$ but with $l_{h}$-the harmonic oscillator length for the hole instead of $l_{e}$, and the energy is $E_{h}=1 / \sigma l_{h}^{2}$, where $\sigma=m_{h} / m_{e}$ is the hole to electron effective mass ratio, or, in other words, the hole mass in the donor units. The negative trion Hamiltonian can be written as

$$
H_{-}=H_{e 1}+H_{e 2}+H_{h}-\frac{1}{r_{e 1 h}}-\frac{1}{r_{e 2 h}}+\frac{1}{r_{12}},
$$

where $r_{e 1 h}\left(r_{e 2 h}\right)$ is the distance between the first (second) electron and the hole and $r_{12}$ is the electron-electron distance. We assume that the lateral confinement is sufficiently large that the trion wave function can be effectively separated into a product

$$
\begin{aligned}
\psi\left(\mathbf{r}_{e 1}, \mathbf{r}_{e 2}, \mathbf{r}_{h}\right)= & \Psi\left(x_{e 1}, y_{e 1}\right) \Psi\left(x_{e 2}, y_{e 2}\right) \Psi\left(x_{h}, y_{h}\right) \\
& \times \chi_{-}\left(z_{e 1}, z_{e 2}, z_{h}\right),
\end{aligned}
$$

where $\chi_{-}$is the negative trion wave function of the motion along the wire. The Hamiltonian (3) integrated over the lateral degrees of freedom with the wave function (4) produces the effective trion Hamiltonian

$$
\begin{aligned}
H_{-}^{\mathrm{ef}}= & -\frac{1}{2}\left(\frac{\partial^{2}}{\partial z_{e 1}^{2}}+\frac{\partial^{2}}{\partial z_{e 2}^{2}}\right)-\frac{1}{2 \sigma} \frac{\partial^{2}}{\partial z_{h}^{2}}+V^{\mathrm{ef}}\left(l_{e} ; z_{e 1}-z_{e 2}\right) \\
& -V^{\mathrm{ef}}\left(l_{e h} ; z_{e 1}-z_{h}\right)-V^{\mathrm{ef}}\left(l_{e h} ; z_{e 2}-z_{h}\right),
\end{aligned}
$$

with $l_{e h}=\sqrt{\left(l_{e}^{2}+l_{h}^{2}\right) / 2}$ and the effective interaction potential $^{9,26}$

$$
V^{\mathrm{ef}}(l ; z)=(\pi / 2)^{1 / 2} \operatorname{erfc}(|z| / \sqrt{2} l) \exp \left(z^{2} / 2 l^{2}\right) / l,
$$

which is finite at the origin $\left[V^{\mathrm{ef}}(l ; 0)=1 / l\right]$ and approaches the $1 / z$ asymptotic at large $z$. Hamiltonian (5) is written with respect to the sum of the ground-state energies of noninteracting two electrons and one hole. Therefore, the absolute value of the (negative) energy of a bound state is interpreted as the energy needed to separate all the particles away from one another. Introducing the center-ofmass coordinate $Z=\left(z_{e 1}+z_{e 2}+\sigma z_{h}\right) /(2+\sigma)$ one obtains $H_{-}^{\mathrm{ef}}=-(1 / 2 M)\left(\partial^{2} / \partial Z^{2}\right)+H_{-}^{\mathrm{rel}}$, where $M=2+\sigma$ is the negative trion mass and $H^{\mathrm{rel}}$ is the relative motion Hamiltonian

$$
\begin{aligned}
H_{-}^{\mathrm{rel}}= & -\frac{1}{2 \mu}\left(\frac{\partial^{2}}{\partial z_{h 1}^{2}}+\frac{\partial^{2}}{\partial z_{h 2}^{2}}\right)-\frac{1}{\sigma} \frac{\partial}{\partial z_{h 1} \partial z_{h 2}}+V^{\mathrm{ef}}\left(l_{e} ; z_{h 1}-z_{h 2}\right) \\
& -V^{\mathrm{ef}}\left(l_{e h} ; z_{h 1}\right)-V^{\mathrm{ef}}\left(l_{e h} ; z_{h 2}\right),
\end{aligned}
$$

with the reduced mass of an electron-hole pair $\mu$ $=\sigma /(1+\sigma)$, and the coordinates of the relative electron-hole positions $z_{h 1}=z_{h}-z_{e 1}$ and $z_{h 2}=z_{h}-z_{e 2}$. In these coordinates the interelectron distance along the length of the wire is $z_{12}=\left|z_{h 1}-z_{h 2}\right|$. The wave function $\chi_{-}$is separable into a product of the center of mass and relative wave function $\chi_{-}\left(z_{e 1}, z_{e 2}, z_{h}\right)=\chi_{C M}(Z) \chi\left(z_{h 1}, z_{h 2}\right)$.

The corresponding relative Hamiltonian for the positive trion has the following form:

$$
\begin{aligned}
H_{+}^{\mathrm{rel}}= & -\frac{1}{2 \mu}\left(\frac{\partial^{2}}{\partial z_{h 1}^{2}}+\frac{\partial^{2}}{\partial z_{h 2}^{2}}\right)-\frac{\partial^{2}}{\partial z_{h 1} \partial z_{h 2}}+V^{\mathrm{ef}}\left(l_{h} ; z_{h 1}-z_{h 2}\right) \\
& -V^{\mathrm{ef}}\left(l_{e h} ; z_{h 1}\right)-V^{\mathrm{ef}}\left(l_{e h} ; z_{h 2}\right),
\end{aligned}
$$

with $z_{h 1}, z_{h 2}$ standing here for the relative position coordinates of the first and second hole with respect to the electron position. The reference energy for the Hamiltonian (8) is the energy of the dissociated complex, i.e., $2 E_{h}+E_{e}$.

In the following we consider also the exciton for which the effective Hamiltonian written with respect to the energy of a dissociated electron and hole pair reads

$$
H^{X}=-\frac{1}{2 \mu} \frac{\partial^{2}}{\partial z_{e h}^{2}}-V^{\mathrm{ef}}\left(l_{e h} ; z_{e h}\right) .
$$

The lowest eigenvalue of this Hamiltonian is equal to minus the exciton binding energy $\left(-E_{B}^{X}\right)$. On the other hand the difference between $-E_{B}^{X}$ and the eigenvalues of trion Hamiltonians [(7) and (8)] is equal to the trion binding energies $\left(E_{B}^{X^{-}}, E_{B}^{X^{+}}\right)$with respect to dissociation into an exciton and a free electron (for $X^{-}$) or a hole (for $X^{+}$). Trion binding energies are equal to the redshift of the trion recombination lines with respect to the exciton line in the PL spectrum. 
We solve the relative Hamiltonian eigenequations using the imaginary time technique ${ }^{27}$ on a two-dimensional grid with a finite-difference approach. We use 201 points in both $z_{h 1}$ and $z_{h 2}$ directions. The size of the computational box in both directions is chosen "self-consistently" to be 12 times larger than the average distance between the particles of the same charge defined as $\left\langle\left(z_{h 1}-z_{h 2}\right)\right\rangle^{1 / 2}$.

In the present calculations we assumed harmonic oscillator lateral confinement which allows us to simplify the problem considerably because of the availability of analytical formula ${ }^{9,26}$ for the effective one-dimensional interaction. The confinement lengths $l_{e}$ and $l_{h}$ parametrize the strength of the particle confinement. Since the single-particle energies cancel in the calculation of the trion binding energies, the applicability of the present results is wider. In fact the present results can be used for any form of the lateral confinement (which does not even have to be cylindrically symmetric) as long as it produces the same effective interaction potential. For instance the electron-electron interaction potential for $l_{e}=2.95$ and $6 \mathrm{~nm}$ is very well (i.e., with a precision better than $2 \%)$ reproduced for a GaAs quantum wire $\left(m_{e}=0.067\right)$ with a circular square well confinement of depth $320 \mathrm{meV}$ and diameters 9.6 and $22.8 \mathrm{~nm}$, respectively. For elliptical harmonic oscillator confinement with different oscillator lengths in $x$ and $y$ directions ( $l_{x}$ and $l_{y}$, respectively) we cannot give a closed analytical formula for the effective interaction potential. Nevertheless, we have found via a numerical integration that the interaction potential between two electrons in an elliptical wire can be surprisingly well reproduced by formula (6) for a circular wire with an effective $l=\left(l_{x}+l_{y}\right) / 2$. The numerically calculated deviation between the two potentials is not larger than $2 \%$ for any interelectron distance. The essential assumption of the present model therefore does not rely on the form of the lateral confinement but on its strength, which has to be large enough to prevent the Coulomb interactions from deforming the lateral wave functions. The applied assumption of the frozen lateral degrees of freedom for the electron and the hole is applicable for the exciton binding energy when $l_{e}<a_{d}$ and $l_{h}<a_{d}$. This condition guarantees that the length of the lateral confinement of the carriers is smaller than the bulk exciton radius, and that the sum of the lateral confinement energies for the electron and the hole are at least two times larger than the exciton binding energy in bulk. For trions the applied approximation is better justified and the conditions are less stringent because the trions have a larger size and have smaller binding energies than the exciton.

\section{RESULTS}

We start the presentation of our results by discussing the properties of trions in quantum wires with equal lateral confinement for the electron and the hole (Sec. III A), and then in Sec. III B we show the effect of different confinements for the stability of $X^{+}$and $X^{-}$trions. Section III C describes the effect of the magnetic field oriented parallel to the axis of the wire.

\section{A. Identical electron and hole lateral confinement}

For equal electron and hole lateral confinement $\left(l_{e}=l_{h}=L\right)$ the electron-electron, the hole-hole, and the
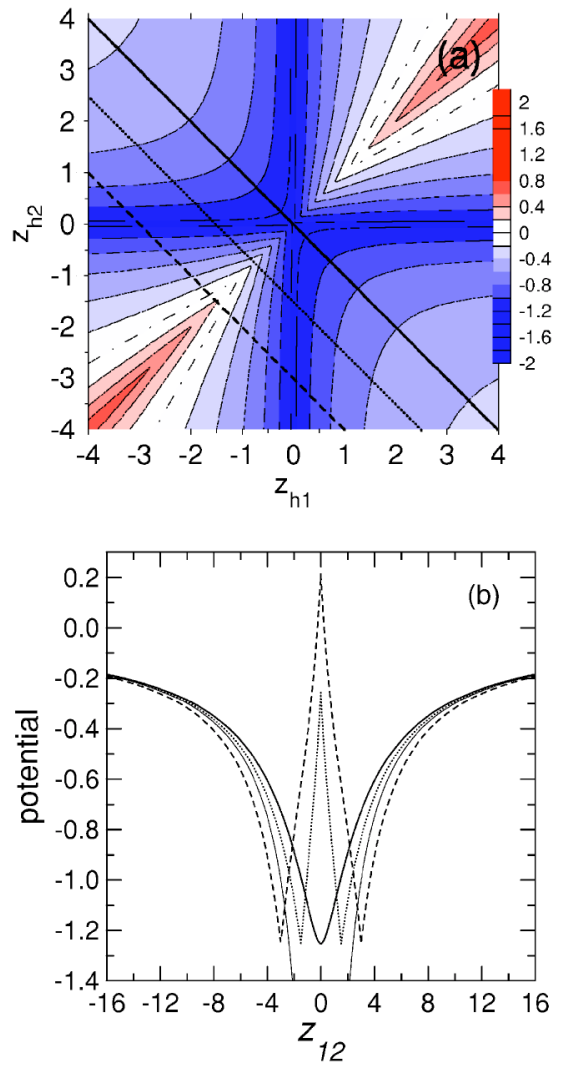

FIG. 1. (a) (Color online) Contour plot of the interaction potential $V=V^{\mathrm{ef}}\left(L ; z_{h 1}-z_{h 2}\right)-V^{\mathrm{ef}}\left(L ; z_{h 1}\right)-V^{\mathrm{ef}}\left(L ; z_{h 2}\right)$ as function of the interparticle distances with lateral confinement length $L=l_{e}=l_{h}=1$. Distances and energies are given in donor units. The dashed-dotted line corresponds to $V=0$. (b) The interaction potential plotted for $L=1$ along the lines $z_{h 2}=-z_{h 1}, z_{h 2}=-z_{h 1}-1.5$, and $z_{h 2}=-z_{h 1}-3$ marked in (a) with (thick) solid, dotted and dashed lines respectively, as function of the interelectron $\left(X^{-}\right)$or interhole $\left(X^{+}\right)$distance $z_{12}=z_{h 1}-z_{h 2}$. Thin solid line shows the $-3 /\left|z_{12}\right|$ asymptotic.

electron-hole interactions have the same form. The total interaction potential, identical for both types of trions, is plotted in Fig. 1(a) as function of $z_{h 1}$ and $z_{h 2}$ for $L=1$. The regions of positive (negative) potentials are plotted with red (blue) colors. Zero of the interaction potential is marked with a dash-dotted line. The interaction potential is minimal along the lines $z_{h 1}=0$ and $z_{h 2}=0$ at which one of the two electrons and the hole are in the same position (for $X^{-}$) or the position of one of the holes coincides with the electron position (for $\left.X^{+}\right)$. The potential is maximal along the diagonal $z_{h 1}=z_{h 2}$ at which the two particles of the same charge are localized in the same point along the wire length. Figure 1(b) shows the cross sections of the interaction potential along the three straight lines in Fig. 1(a) as function of the interelectron $\left(X^{-}\right)$ or interhole $\left(X^{+}\right)$distance $z_{12}$. On the antidiagonal the interaction has the form of a triangular potential well [cf. solid line in Fig. 1(b)]. Moving along the antidiagonal is equivalent to interchange the position of the two particles of the same charge with fixed position of the third particle of the opposite charge. Along the paths plotted with dotted and dashed lines in Fig. 1(a), which are shifted below the antidiagonal, the potential has the form of a double potential well 

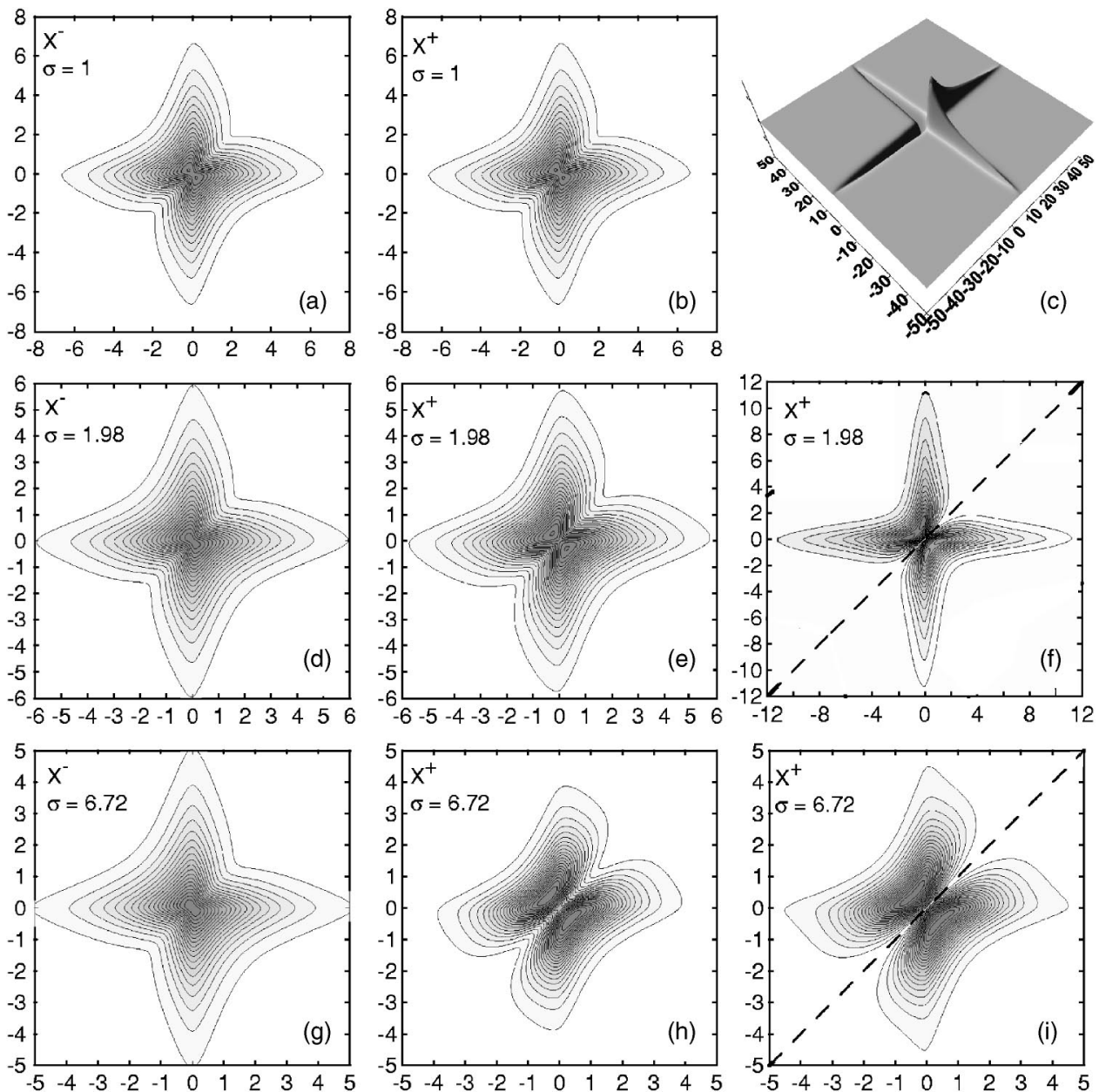

FIG. 2. Wave functions for negative (a,d,g,j) and positive (b,c,e,f,h,i,k,l) trions for $l_{e}=l_{h}=L$ $=0.2$ in $z_{h 1}$ and $z_{h 2}$ coordinates (horizontal and vertical axis, respectively) for different values of the mass ratio $\sigma$. Plots $(\mathrm{c}, \mathrm{f}, \mathrm{i}, \mathrm{l})$ show the wave functions of the excited $X^{+}$state antisymmetric with respect to the interchange of the holes. The dashed line in (f,i,l) shows the node of the wave functions. Plot (c) corresponds to an unbound state, for other plots the computational box is larger than the fragment displayed and the states are bound.
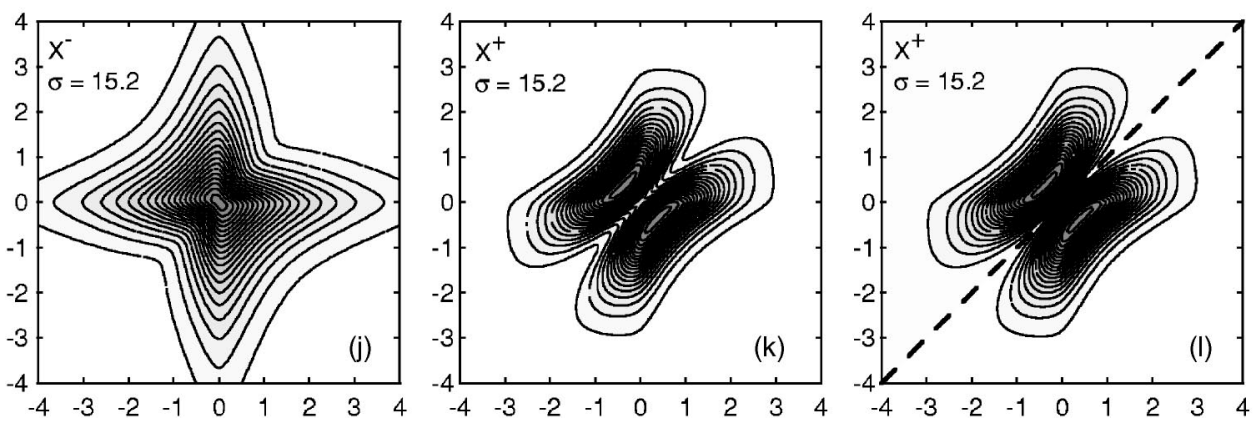

[see Fig. 1(b)] with a barrier near the diagonal resulting from the repulsion of the equally charged particles. For large $z_{12}$ the potential approaches $-3 / z_{12}$ asymptotically which is shown by the solid line in Fig. 1(b).

Contour plots of the wave function of the negative and positive trions calculated for different effective mass ratios are plotted in Fig. 2 for $L=0.2$. For $l_{e}=l_{h}$ the negative and positive trion relative Hamiltonians (7) and (8) differ only by the factor standing in front of the mixed derivative $(1 / \sigma$ for $X^{-}$and 1 for $X^{+}$). The ground-state wave function for $\sigma=1$ is the same for both trions [cf. Figs. 2(a) and 2(b)]. The first excited state of $X^{+}$, which is antisymmetric with respect to the interchange of the holes is unbound. Its wave function calculated for the size of the computational box $100 \times 100$ (in donor Bohr radius units) is plotted in Fig. 2(c). For the unbound state no computational box is large enough (i.e., the

wave function vanishes only at the ends of the computational box). The wave function is nonzero only near both axis. One of the holes stays at the position of the electron and the other strives to be as far as possible from the other two particles.

Results for $\sigma=1.98$ plotted in Figs. 2(d)-2(f) correspond to $\mathrm{CdTe}$ material parameters $\left(m_{h}=0.19 m_{0}, m_{e}=0.096 m_{0}\right)$ with the donor units $a_{d}=5.4 \mathrm{~nm}$ and $2 R_{d}=27.6 \mathrm{meV}$. The probability density maximum for $\sigma=1$ is split into two extrema at the antidiagonal of the plots [cf. Figs. 2(a) and 2(b)]. For $\sigma>1$ these two extrema merge into a single one for $X^{-}$ [see Fig. 2(d)] and for $X^{+}$they become more distinctly separated [see Fig. 2(e)] and the excited state for $X^{+}$becomes bound [cf. Fig. 2(f)].

Figures 2(g)-2(i) and 2(j)-2(1) for $\sigma=6.7$ and $\sigma=15.2$ correspond to GaAs $\left(m_{h}=0.45 m_{0}, m_{e}=0.067 m_{0}\right.$, $\left.2 R_{d}=11.9 \mathrm{meV}, \quad a_{d}=9.8 \mathrm{~nm}\right)$ and InAs $\left(m_{h}=0.41 m_{0}\right.$, 


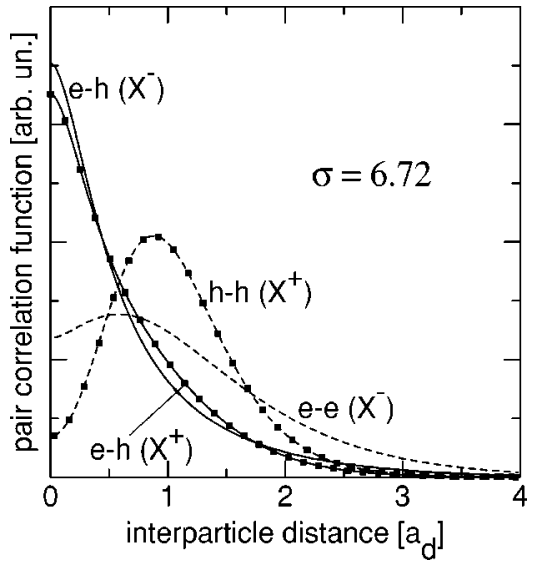

FIG. 3. Electron-hole (solid lines), electron-electron, hole-hole (dashed lines) pair correlation functions plots for $X^{-}$and $X^{+}$(lines marked by black squares) at $\sigma=6.72$ and $l_{e}=l_{h}=0.2$.

$m_{e}=0.027 m_{0}, 2 R_{d}=3.2 \mathrm{meV}$, and $\left.a_{d}=29.7 \mathrm{~nm}\right)$ material parameters, respectively. Increasing $\sigma$ has an opposite effect on the $X^{-}$and $X^{+}$wave functions. For the negative (positive) trion the local minimum along the diagonal $z_{h 1}=z_{h 2}$ is less (more) pronounced. The wave function evolution with $\sigma$ is related to the tunneling of the particles of the same charge via the potential barrier presented in Fig. 1(b). The electrons in $X^{-}$with light effective masses tunnel easily through the diagonal barrier due to the interelectron repulsion. On the other hand the diagonal barrier is effectively much larger for the heavy-mass holes which prevents its penetratation at large $\sigma$ which leads to the appearance of the characteristic maxima elongated along the diagonal in Figs. 2(e), 2(h), and 2(k).

The correlation between the particles in the complexes is more clearly visible in the pair correlation functions plotted in Fig. 3. The electron-hole correlation function is calculated as $f_{e h}(z)=\int d z_{h 1} d z_{h 2}\left|\chi\left(z_{h 1}, z_{h 2}\right)\right|^{2} \delta\left(z-z_{h 1}\right)$ and the hole-hole (for $X^{+}$), and electron-electron (for $X^{-}$) as $f_{\text {same }}(z)=\int d z_{h 1} d z_{h 2}\left|\chi\left(z_{h 1}, z_{h 2}\right)\right|^{2} \delta\left[z-\left(z_{h 1}-z_{h 2}\right)\right]$. The Coulomb hole in the hole-hole correlation in $X^{+}$is much larger than for electrons in $X^{-}$but at the expense of slightly weaker relative electron-hole localization.

The binding energies of the exciton and the trions for $L=0.2$ are plotted as functions of $\sigma$ in Fig. 4. All the binding energies are increasing functions of $\sigma$. In bulk the first excited state of the positive trion is antisymmetric with respect to the hole interchange, ${ }^{28}$ possess the $P$ symmetry and is bound for $\sigma>4.2$. The critical value of the mass ratio is much smaller for quasi-one-dimensional (1D) confinement. ${ }^{9}$ Here, for $L=0.2$ the excited $X^{+}$state is bound for $\sigma>1.2$ (see Fig. 3). For quasi-1D confinement the lowest excited state has the $S$ symmetry with respect to the axis of the wire but is of odd spatial parity, i.e., it is antisymmetric with respect to simultaneous change of sign of all the $z$ coordinates (see Fig. 2 ). The ground state of $X^{+}$becomes degenerate with respect to the symmetry of the wave function, i.e., the hole interchange, for large $\sigma$ for which tunneling through the diagonal potential barrier (cf. Fig. 1) disappears. At large $\sigma$ also the probability density of the excited $X^{+}$level becomes identical to the ground-state probability density (cf. Fig. 2). $X^{-}$does not possess a bound excited state for $\sigma>1$.

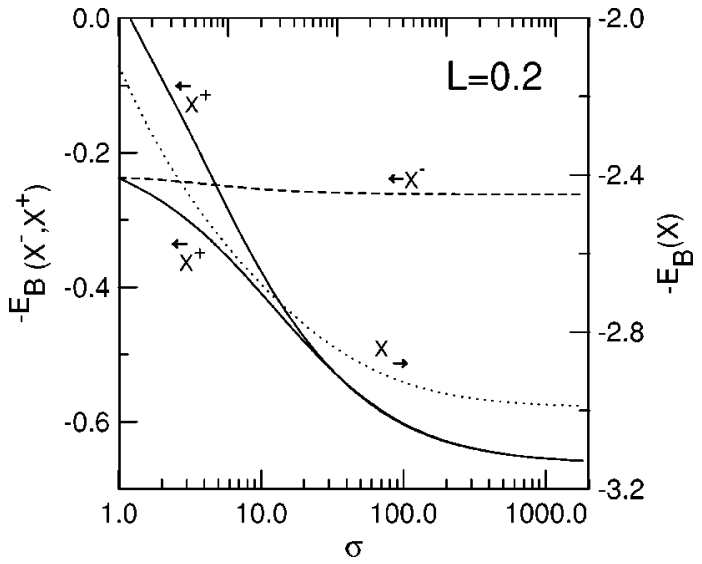

FIG. 4. Binding energies of the negative (dashed lines) and positive trion (solid lines) states for $L=0.2$ as function of the mass ratio $\sigma$. Higher solid curve corresponds to the $X^{+}$state antisymmetric with respect to the interchange of electrons and holes, i.e., it is the first excited state. Thin vertical lines show the values of $\sigma=1,1.98$, 6.72, and 15.2. Dotted curve, referred to the right axis, shows the exciton ground-state eigenvalue. Energies and lengths are in donor units.

The inset of Fig. 5 shows the ground-state energy for $\sigma=15.2$ as function of the lateral confinement length. In the $L=0$ limit the average interparticle distances decrease to zero and the energies diverge to minus infinity. This is a consequence of the Coulomb interaction singularity in one dimension. ${ }^{29}$ The main part of Fig. 5 shows the shifts of the trion PL lines with respect to the exciton line (calculated as the difference of the eigenvalues presented in the inset) for different values of $\sigma$. It turns out that the binding energies have a power law dependence on $L$, i.e., $L^{-q}$, for the $X^{-}$and

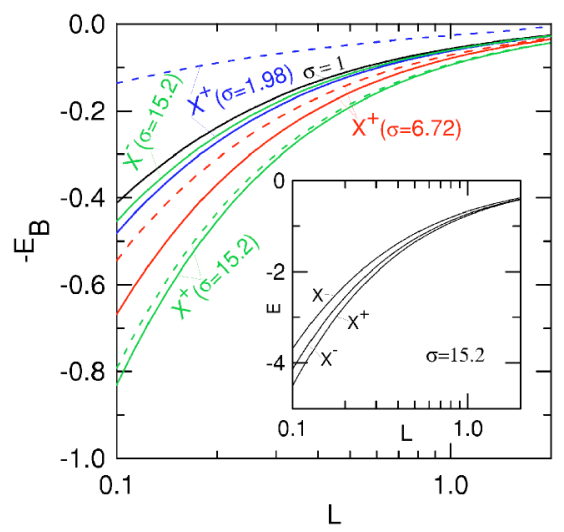

FIG. 5. (Color online) Binding energies of the trions as functions of the length of the lateral confinement. Lines for $\sigma=1,1.98$, 6.72 , and 15.2 plotted with black, blue, red, and green colors, respectively. For $\sigma=1$ binding energies of $X^{-}$and $X^{+}$are equal. The dashed lines are the energies of the excited $X^{+}$states antisymmetric with respect to the interchange of the holes $\left(X^{-}\right.$does not possesses a bound excited state for $\sigma>1$ ). Lines for $X^{-}$at $\sigma=1.98$ and 6.72 have been omitted for clarity-they are situated between the $\sigma=1$ line and $\sigma=15.2$ line for $X^{-}$(see Fig. 4). Inset: energy eigenvalues for neutral exciton and charged trions for $\sigma=15.2$. Energies and lengths are given in donor units. 


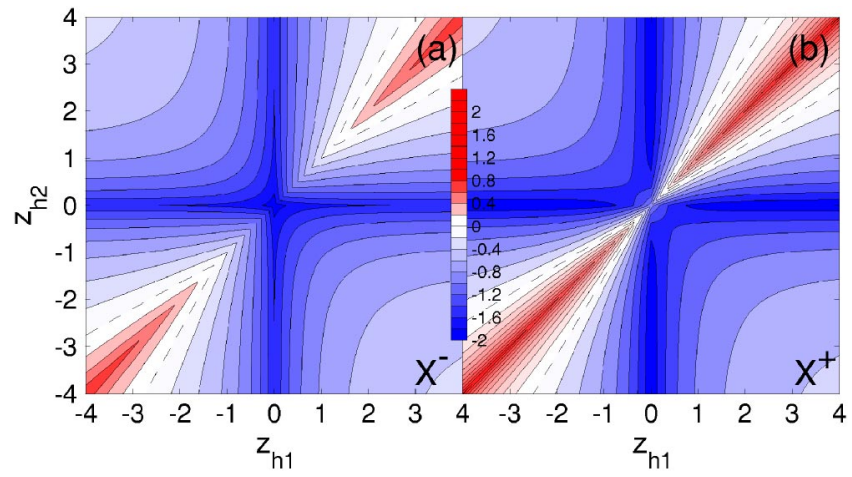

FIG. 6. (Color online) Contour plot of the interaction potential for (a) negative trion $V=V^{\mathrm{ef}}\left(l_{e} ; z_{h 1}-z_{h 2}\right)-V^{\mathrm{ef}}\left(l_{e h} ; z_{h 1}\right)-V^{\mathrm{ef}}\left(l_{e h} ; z_{h 2}\right)$ and (b) positive trion $V=V^{\mathrm{ef}}\left(l_{h} ; z_{h 1}-z_{h 2}\right)-V^{\mathrm{ef}}\left(l_{e h} ; z_{h 1}\right)-V^{\mathrm{ef}}\left(l_{e h} ; z_{h 2}\right)$ as function of the interparticle distances for the lateral confinement lengths $l_{e}=1$ and $l_{h}=0.5$. Distances and energies are given in donor units.

$X^{+}$ground state presented in this figure $q$ changes from 0.83 $(\sigma=1)$ to $0.91\left(X^{+}\right.$for $\left.\sigma=15.2\right)$.

\section{B. Effect of different electron and hole lateral confinement}

Let us now consider the interaction potential for stronger hole confinement. Figures 6(a) and 6(b) show the interaction potentials for $l_{e}=1$ as in Fig. 1 but for smaller $l_{h}=0.5$. For both the negative [cf. Fig. 6(a)] and the positive trion [cf. Fig. 6(b)] the potential minima at $z_{h 1}=0$ and $z_{h 2}=0$ become deeper with respect to the $l_{e}=l_{h}$ case presented in Fig. 1. For $X^{-}$the electron-electron interaction (the diagonal potential barrier) is not affected by the change of $l_{h}$ [compare Figs. 1 and $6(\mathrm{a})]$. On the other hand the hole-hole repulsive interaction for $X^{+}$is strongly increased.

The effect of the hole confinement on the trion binding energies is plotted in Fig. 7 for GaAs material parameters and fixed values of the electron lateral confinement. Consistently with the results of Sec. III A for $l_{e}=l_{h}$ the positive trion is more stable than the negative trion. A decrease of $l_{h}$ below the value of $l_{e}$ results in the interchange of the $X^{-}$and

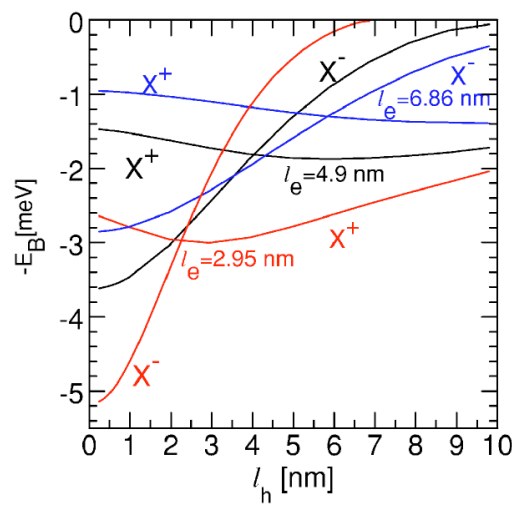

FIG. 7. (Color online) Shifts of the trion recombination PL lines with respect to the PL exciton line as function of the hole confinement length $\left(l_{h}\right)$ for GaAs. Different values of the electron confinement length are plotted with different colors.

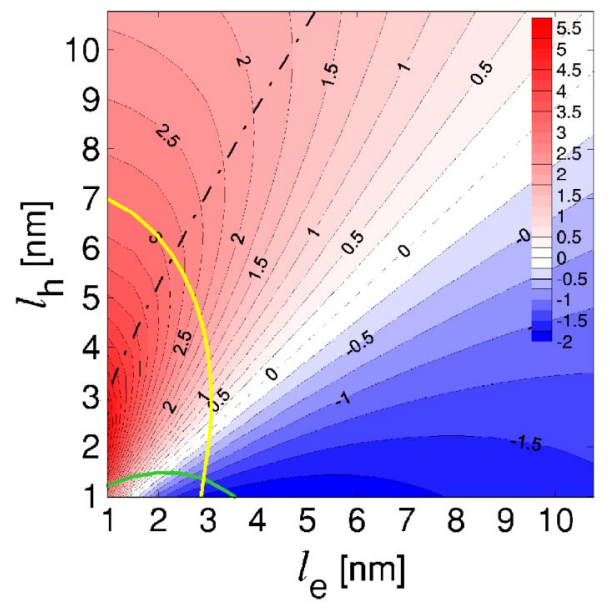

FIG. 8. (Color online) Difference of the positive and negative trion binding energies (in milli-electron-volts) as function of the electron and hole confinement lengths for GaAs material parameters. Blue (red) regions correspond to more stable negative (positive) trion. Above the dashed-dotted line the negative trion is unbound. The green line corresponds to $E_{B}\left(X^{-}\right)=4.2 \mathrm{meV}$ and the yellow line to $E_{B}\left(X^{+}\right)=2.9 \mathrm{meV}$.

$X^{+}$energy lines. ${ }^{30}$ This is due to the enhanced hole-hole interaction shown in Fig. 6(b). The negative trion binding energy is a monotonous function of the hole confinement length, the larger $l_{h}$ the smaller is the electron-hole interaction stabilizing $X^{-}$. The situation is more complex for $X^{+}$, since with increasing $l_{h}$ also the destabilizing hole-hole interaction decreases. As a consequence the positive trion binding energy possesses a maximum as function of $l_{h}$.

The difference of the positive and negative trion binding energies is plotted in Fig. 8. Both the trions are equally stable for $l_{h}=0.92 l_{e}-0.38 \mathrm{~nm}$. For $l_{h}$ larger (smaller) than $0.92 l_{e}-0.38{\mathrm{~nm} X^{+}}$is more (less) stable than $X^{-}$. The maximum of the $X^{+}$binding energy presented in Fig. 7 follows a path that is nearly linear for $l_{e}>2 \mathrm{~nm}$ and is approximately parametrized by $l_{h}=1.62 l_{e}-1.98 \mathrm{~nm}$. For the points at the left of the dash-dotted line the electron-hole attractive interaction stabilizing the trion is so weak with respect to the electron-electron repulsive interaction destabilizing the complex that the negative trion stops to be bound (see also the line for $X^{-}$at $l_{e}=2.95 \mathrm{~nm}$ in Fig. 7). The absence of the negative trion binding requires a substantially weaker hole confinement than the confinement of the electron which is rather impossible to obtain in the presently produced quantum wires and would require the valence band offset between the wire and the matrix to be much smaller than the conduction band offset. Moreover, the present modeling based on the assumption that the lateral wave functions are not affected by the interaction is likely to fail since the hole wave function is very likely to become more localized due to the attraction by strongly confined electrons.

The fit of the calculated $X^{-}$and $X^{+}$binding energies to the experimental data is obtained at the crossing of the green and yellow lines, i.e., for $l_{e}=2.95 \mathrm{~nm}$ and $l_{h}=1.3 \mathrm{~nm}$. The obtained fit corresponds to realistic values which give a general idea on the particle confinement in the wire (the measurements ${ }^{11}$ were performed on a V-groove 


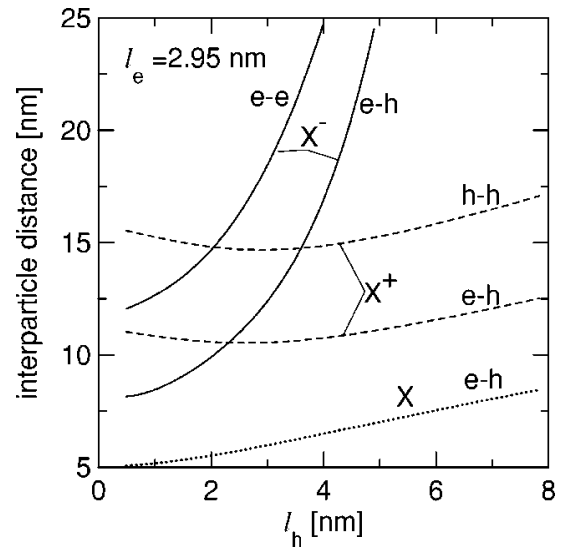

FIG. 9. Interparticle distances for the negative (solid lines) and positive trions (dashed lines) and for the exciton (dotted line) for GaAs material parameters and $l_{e}=2.95 \mathrm{~nm}$. $(e-e),(e-h)$, and $(h-h)$ stand for the electron-electron, the electron-hole and the hole-hole distance.

GaAs/AlGaAs quantum wire with a thickness of the GaAs crescent of $3 \mathrm{~nm}$ at the center). Obviously, a more realistic model is required to extract details of the confinement from the experimental data.

The dependence of the size of the trion, i.e., the interparticle distance as function of the hole confinement length, is shown in Fig. 9 for $l_{e}=2.95 \mathrm{~nm}$. The electron-hole distance for the trions and the exciton have been calculated as $\sqrt{\left\langle z_{h 1}^{2}\right\rangle}$, and $\sqrt{\left\langle z_{e h}^{2}\right\rangle}$, respectively. The hole-hole distance for $X^{+}$and the electron-electron distance for $X^{-}$are determined as $\sqrt{\left\langle\left(z_{h 1}-z_{h 2}\right)^{2}\right\rangle}$. The size of the exciton increases as $l_{h}$ increases which is due to the reduced value of the electron-hole interaction. Much stronger dependence on $l_{h}$ is observed for $X^{-}$, which becomes unbound for $l_{h}>7 \mathrm{~nm}$ (cf. Fig. 7). The dependence of the $X^{+}$size on the hole confinement is nonmonotonous. The positively charged complex has the smallest size near $l_{h}=3 \mathrm{~nm}$ when it is the most strongly bound (cf. Fig. 7). For $l_{h}=l_{e}=2.95 \mathrm{~nm}$ the order of the interparticle distances in the two complexes is the same as in twodimensional quantum wells (compare Fig. 4 of Ref. 13). In spite of the fact that the probability of finding both holes in $X^{+}$in the same position is much smaller than for electrons in $X^{-}$(cf. Fig. 3) the longer tail of the electron-electron correlation function results in a larger electron-electron distance than hole-hole distance.

\section{Magnetic field parallel to the wire}

In the present approach it is straightforward to include a magnetic field oriented parallel to the axis of the wire. It simply scales down the electron and hole oscillator lengths according to the formula $l(B)=\left[1 / l^{4}(0)+1 / l_{c}^{4}\right]^{-1 / 4}$, where $l_{c}=\sqrt{2 \hbar / e B}$ is the magnetic field length $\left(l_{c}=36.28 / \sqrt{B} \mathrm{~nm} \sqrt{T}\right)$. Since $l(B)$ for high magnetic fields decreases to $l_{c}$, the magnetic field tends to equalize the electron and hole confinement. The binding energy of the trions can be obtained following paths of $\left[l_{h}(B), l_{e}(B)\right]$ in Fig. 8 . The modification of the binding energies of the trions by the

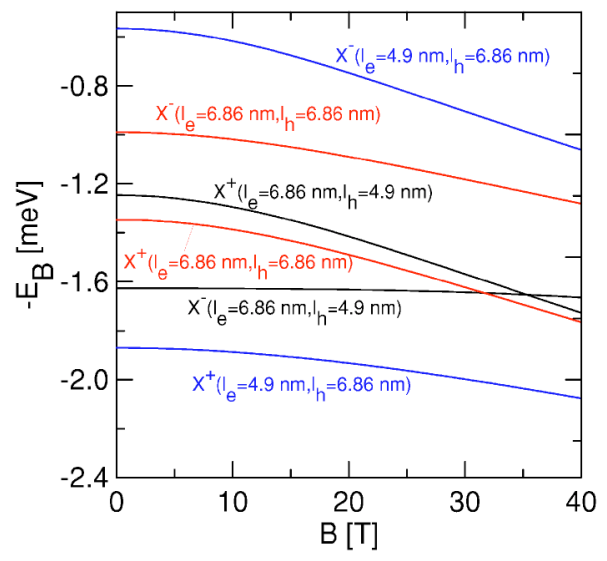

FIG. 10. (Color online) Magnetic field dependence of the trion binding energies in GaAs for different values of the electron and hole oscillator lengths.

magnetic field is presented in Fig. 10 for different oscillator lengths. In a magnetic field of $40 \mathrm{~T}, l(B=0)=4.9 \mathrm{~nm}$ is decreased to $l(B=40 \mathrm{~T})=4.4 \mathrm{~nm}$ and $l(B=0)=6.86 \mathrm{~nm}$ to $l(B=40 \mathrm{~T})=5.19 \mathrm{~nm}$. For $l_{e}=l_{h}=6.86 \mathrm{~nm}$ (see the red curves in Fig. 10) the magnetic field decreases the length of confinement exactly as in the case presented in Fig. 5. In the more realistic case of stronger hole confinement, i.e., for $l_{e}=6.86 \mathrm{~nm}$ and $l_{h}=4.9 \mathrm{~nm}$ (see the black lines in Fig. 10) the magnetic field increases the binding energy of the positive trion more strongly. This is because the magnetic field more strongly affects the larger $l_{e}$ value than the smaller hole confinement length $l_{h}$ which increases the electron-hole interaction more strongly than the repulsive hole-hole potential. For $X^{-}$the effect of the increased electron-hole interaction is nearly cancelled by the increase of the electronelectron potential value. Near $35 \mathrm{~T}$ a crossing of the black lines is observed, which corresponds to passing from the "blue" to the "red" region in the phase diagram of Fig. 8. This crossing is qualitatively opposite to the one obtained for two-dimensional quantum wells, ${ }^{13}$ in which a small magnetic field (around $1 \mathrm{~T}$ ) increased the $X^{-}$stability over the $X^{+}$ binding energy. In quantum wells the magnetic-field related increase of the single-particle energy is smaller for electrons and holes bound in the trion complex than for the lowest Landau level in the final state of the free electron and hole after the trion dissociation. The crossing of the binding energies observed in quantum wires ${ }^{13}$ is therefore at least partially due to the stronger dependence of the electron lowest Landau level—note an almost linear magnetic field dependence of the trion binding energies in Fig. 2 of Ref. 13. In the present calculations the free electron and the free hole are strongly localized in the plane perpendicular to the field and the single-particle magnetic field effects cancel due to the assumption of the frozen-lateral degrees of freedom, so that the crossing is entirely due to the modified effective interparticle interactions.

In the case of stronger electron confinement $\left(l_{e}=4.9 \mathrm{~nm}, l_{h}=6.86 \mathrm{~nm}\right.$ - the blue lines in Fig. 10) the situation is just opposite, the $X^{-}$is less strongly bound and the magnetic field acts more strongly on the negative trion decreasing the binding energy difference according to the 
mechanism described above. However, for the electron confinement equal or stronger than the hole confinement, the magnetic field does not lead to crossing of the trion energy lines and $X^{+}$is more stable for any magnetic field.

\section{SUMMARY AND CONCLUSIONS}

We studied the properties of the negative and positive trions in quantum wires with strong lateral confinement using the approximation of the lowest subband occupancy which allows for a numerically exact solution of the multiparticle Schrödinger equation. We investigated the relative stability of the positive and negative trions with respect to the dissociation into an exciton and a free carrier for different electron and hole confinement. We found that the order of the negative and positive trion PL lines is interchanged when the lateral confinement of the hole is stronger than the one for the electron. In a GaAs quantum wire with $l_{e}=5 \mathrm{~nm}$ we predict that when $l_{h}$ is $20 \%$ smaller the positive and negative trion recombination lines interchange. The change in the order is due to modification of the effective interactions in the trion complexes. The present results provide an explanation for the recently experimentally observed larger stability of the negative trion in quantum wires. ${ }^{11} \mathrm{We}$ predict that for larger $X^{-}$stability due to stronger hole confinement the magnetic field oriented parallel to the axis will tend to interchange the order of the $X^{+}$and $X^{-}$energy lines.

\section{ACKNOWLEDGMENTS}

This paper was supported by the Flemish Science Foundation (FWO-Vl), the Belgian Science Policy, the University of Antwerpen (VIS and GOA), the EU-NoE SANDiE, and by the Polish Ministry of Scientific Research and Information Technology within the solicited Grant No. PBZ-MENMIN-008/P03/2003. B.S. is supported by the EC Marie Curie IEF Project No. MEIF-CT-2004-500157. We are thankful to Daniel Oberli, Bart Partoens, and Yosip Sidor for helpful discussions.
${ }^{1}$ M. A. Lampert, Phys. Rev. Lett. 1, 450 (1958).

${ }^{2}$ B. Stébé and A. Ainane, Superlattices Microstruct. 5, 545 (1989).

${ }^{3}$ C. Riva, F. M. Peeters, and K. Varga, Phys. Rev. B 61, 13873 (2000).

${ }^{4}$ B. Stébé, A. Moradi, and F. Dujardin, Phys. Rev. B 61, 7231 (2000).

${ }^{5}$ S. Glasberg, G. Finkelstein, H. Shtrikman, and I. Bar-Joseph, Phys. Rev. B 59, R10425 (1999).

${ }^{6}$ A. Wójs and P. Hawrylak, Phys. Rev. B 51, 10880 (1995).

${ }^{7}$ D. M. Whittaker and A. J. Shields, Phys. Rev. B 56, 15185 (1997).

${ }^{8}$ J. R. Chapman, N. F. Johnson, and V. N. Nicopoulos, Phys. Rev. B 55, R10221 (1997).

${ }^{9}$ A. Esser, R. Zimmermann, and E. Runge, Phys. Status Solidi B 227, 317 (2001).

${ }^{10}$ T. Tsuchiya, Int. J. Mod. Phys. B 15, 3985 (2001).

${ }^{11}$ T. Otterburg, D. Y. Oberli, M.-A. Dupertois, N. Moret, E. Pelucchi, B. Dwir, K. Leifer, and E. Kapon, cond-mat/0410050 (unpublished).

${ }^{12}$ B. Stébé and G. Munschy, Solid State Commun. 17, 1051 (1975).

${ }^{13}$ C. Riva, F. M. Peeters, and K. Varga, Phys. Rev. B 64, 235301 (2001).

${ }^{14}$ G. Yusa, H. Shtrikman, and I. Bar-Joseph, Phys. Rev. Lett. 87, 216402 (2001).

${ }^{15}$ A. V. Filinov, C. Riva, F. M. Peeters, Yu. E. Lozovik, and M. Bonitz, Phys. Rev. B 70, 035323 (2004).

${ }^{16}$ B. Szafran, B. Stébé, J. Adamowski, and S. Bednarek, J. Phys.:
Condens. Matter 12, 2453 (2000).

${ }^{17}$ B. Szafran, B. Stébé, J. Adamowski, and S. Bednarek, Phys. Rev. B 66, 165331 (2002).

${ }^{18}$ F. Guffarth, S. Rodt, A. Schliwa, K. Pötschke, and D. Bimberg, Physica E (Amsterdam) 25, 261 (2004).

${ }^{19}$ D. Bellucci, F. Troiani, G. Goldoni, and E. Molinari, Phys. Rev. B 70, 205332 (2004).

${ }^{20}$ F. Rossi, G. Goldoni, and E. Molinari, Phys. Rev. Lett. 78, 3527 (1997).

${ }^{21}$ J. Maes, M. Hayne, Y. Sidor, B. Partoens, F. M. Peeters, Y. González, L. González, D. Fuster, J. M. García, and V. V. Moshchalkov, Phys. Rev. B 70, 155311 (2004).

${ }^{22}$ A. Gustafsson, F. Reinhardt, G. Biasiol, and E. Kapon, Appl. Phys. Lett. 67, 3673 (1995).

${ }^{23}$ L. González, J. M. García, R. García, F. Briones, J. MartínezPastor, and C. Ballesteros, Appl. Phys. Lett. 76, 1104 (2000).

${ }^{24}$ T. W. Kim, E. H. Lee, K. H. Lee, J. S. Kim, and H. L. Park, Appl. Phys. Lett. 83, 4235 (2003).

${ }^{25}$ J.-B. Xia and K. W. Cheah, Phys. Rev. B 55, 1596 (1997).

${ }^{26}$ S. Bednarek, B. Szafran, T. Chwiej, and J. Adamowski, Phys. Rev. B 68, 045328 (2003).

${ }^{27}$ K. T. Davies, H. Flocard, S. Kreger, and M. S. Weiss, Nucl. Phys. A 342, 112 (1980).

${ }^{28}$ A. M. Frolov and A. Yu. Yeremin, J. Phys. B 22, 1263 (1989).

${ }^{29}$ R. Loudon, Am. J. Phys. 27, 649 (1959).

${ }^{30}$ The crossing point occurs exactly at $l_{e}=l_{h}$ only for $\sigma=1$. 